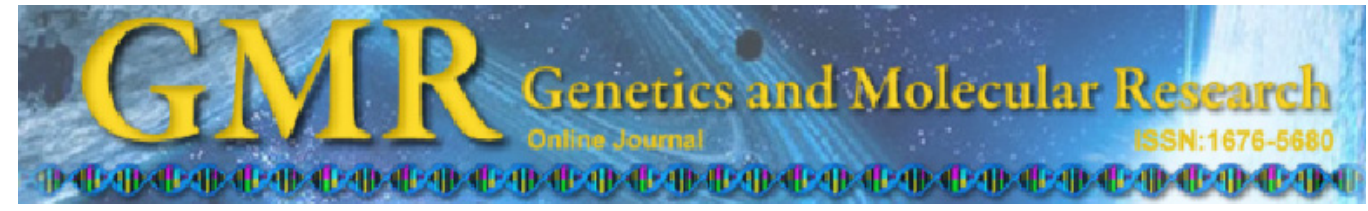

\title{
Improved thermostable $\alpha$-amylase activity of Bacillus amyloliquefaciens by low-energy ion implantation
}

\author{
X.Y. Li, J.L. Zhang and S.W. Zhu \\ Key Laboratory of Crop Biology of Anhui Province, School of Life Science, \\ Anhui Agricultural University, Hefei, China \\ Corresponding author: S.W. Zhu \\ E-mail: zhusuwen@126.com
}

Genet. Mol. Res. 10 (3): 2181-2189 (2011)

Received September 27, 2010

Accepted August 13, 2011

Published September 23, 2011

DOI http://dx.doi.org/10.4238/vol10-3gmr1081

\begin{abstract}
Thermostable $\alpha$-amylase is of great importance in the starch fermentation industry; it is extensively used in the manufacture of beverages, baby foods, medicines, and pharmaceuticals. Bacillus amyloliquefaciens produces thermostable $\alpha$-amylase; however, production of thermostable $\alpha$-amylase is limited. Ion-beam implantation is an effective method for mutation breeding in microbes. We conducted ionbeam implantation experiments using two different ions, $\mathrm{Ar}^{+}$and $\mathrm{N}^{+}$, to determine the survival rate of and dose effect on a high $\alpha$-amylase activity strain of $B$. amyloliquefaciens that had been isolated from soil samples. $\mathrm{N}^{+}$implantation resulted in a higher survival rate than $\mathrm{Ar}^{+}$ implantation. The optimum implantation dose was $2.08 \times 10^{15}$ ions/ $\mathrm{cm}^{2}$. Under this implantation condition, we obtained a thermally and genetically stable mutant $\alpha$-amylase strain (RL-1) with high enzyme activity for degrading $\alpha$-amylase. Compared to the parental strain (RL), the RL-1 strain had a $57.1 \%$ increase in $\alpha$-amylase activity. We conclude that ion implantation in B. amyloliquefaciens can produce strains with increased production of thermostable $\alpha$-amylase.
\end{abstract}

Key words: Ion implantation; Bacillus amyloliquefaciens; Thermostable $\alpha$-amylase 


\section{INTRODUCTION}

Amylases are enzymes capable of hydrolyzing starch and are produced by animals, plants, and microbes (Hoj et al., 1989). These enzymes can specifically cleave the $O$-glycosidic bonds in starch, a storage polysaccharide present in the seeds and tubers of various plants. Starch is composed of $\alpha-1,4$-linked $\alpha$-D-glucose units from amylose and amylopectin; these starch components differ from each other in the degree of branching. $\alpha$-Amylase is important in the metabolism of maltose and maltodextrins. The potential for commercial application of $\alpha$-amylases is enormous, and its preparation accounts for approximately $25 \%$ of the enzyme production market (Azad et al., 2009).

Starch and glycogen are the most commonly used organic substrates in the fermentation industry. Currently, these substrates are largely degraded chemically, and the cost of this process is very high. Degradation of starch and glycogen using microbial fermentation (mostly at high temperatures) ensures optimal chemical degradation, low cost, and environmental protection. Because the thermostable $\alpha$-amylase excreted by Bacillus amyloliquefaciens has considerably high thermal stability, it has been widely used in the production of beer, alcohol, foods, etc., since its isolation in 1973 (Tee and Kaletunc, 2009). However, very few natural strains produce thermostable $\alpha$-amylases that can be used for the degradation of starch and glycogen. This feature of the natural strains is mainly attributed to the low enzyme activity of the $\alpha$-amylases they produce.

Genetic variation in amylase activity and inducibility has been analyzed in Drosophila melanogaster (Yamate and Yamazaki, 1999). In fungi, there are 2 major classes of starch-degrading enzymes: $\alpha$-amylases and glucoamylases. Aspergillus awamori and A. oryzae produce glucoamylases that can absorb and digest raw starch. In addition, the raw starch-degrading activity of Rhizopus niveus glucoamylase is stronger than that of Aspergillus sp glucoamylase (Fielden et al., 2002). However, little is known about the adaptation of enzymes other than glucoamylase in a starch-rich environment.

Previous studies on starch-degrading strains have mainly focused on the enzymatic properties and conditions of fermentation (Okita et al., 1979). Additionally, many studies on cloning and expression of thermostable $\alpha$-amylases use genetic engineering and molecular microbiology techniques (Dong et al., 2008). Therefore, screening and improving the outstanding strains via new technology and/or new methods to produce a thermostable $\alpha$-amylase that use starch more efficiently are intriguing prospects (Mollania et al., 2010).

The technique of ion-beam genetic modification has been widely applied in recent years. Previous research has shown that low-energy ion implantation into solid biological material has a unique mode of action. Thus, low-energy ion beams have many applications in the life sciences. During the 1980s, a scientific research group at the Institute of Plasma Physics, Chinese Academy of Science, first applied low-energy ion implantation to modify microorganisms (Xie et al., 2003). After years of development, low-energy ion implantation is now being widely used as a technique for inducing mutations in plants and microorganisms (Li et al., 2009), but few studies have reported the use of this technique to induce mutations in B. amyloliquefaciens. Thus, in this study, low-energy ion implantation was used to irradiate a $B$. amyloliquefaciens strain to screen for mutants producing highly active and thermostable $\alpha$-amylase. We successfully isolated such a mutant. Here, we provide experimental evidence supporting the use of this mutant strain for efficient degradation of starch and glycogen. 


\section{MATERIAL AND METHODS}

\section{Bacterial strain}

The RL strain with highly thermostable amylase activity was isolated from 100 soil samples. We collected the 100 soil samples from areas with high temperature, such as hot springs, flour mills, and breweries, in Anhui Province, China. The RL strain displayed amylase activity at high temperatures $\left(70^{\circ} \mathrm{C}\right)$ and was identified as $B$. amyloliquefaciens on the basis of physiological and morphological analysis and $16 \mathrm{~S}$ rDNA sequencing.

\section{Culture media}

The preliminary screening culture medium $(1 \mathrm{~L})$ contained $2.0 \mathrm{~g}$ soluble starch, 1.0 g peptone, $0.5 \mathrm{~g} \mathrm{NaCl}$, and $2.0 \mathrm{~g}$ agar. The slant medium (1 L; $\mathrm{pH}$ 6.0-7.0) contained $0.5 \mathrm{~g}$ $\mathrm{NaCl}, 0.5 \mathrm{~g}$ beef extract, $0.8 \mathrm{~g}$ maltose, $0.5 \mathrm{~g}$ soluble starch, and $1.8 \mathrm{~g}$ agar. The shaking flask culture medium ( $1 \mathrm{~L} ; \mathrm{pH} 7.0$ ) contained $2.0 \mathrm{~g}$ soluble starch, $1.0 \mathrm{~g}$ peptone, $0.3 \mathrm{~g}$ beef extract, and $0.5 \mathrm{~g} \mathrm{NaCl}$. The enrichment culture medium $(1 \mathrm{~L} ; \mathrm{pH} 7.2-7.5)$ consisted of $1.0 \mathrm{~g}$ peptone, $0.5 \mathrm{~g}$ beef extract, $0.5 \mathrm{~g} \mathrm{NaCl}$, and $0.5 \mathrm{~g} \mathrm{CaCl}_{2}$. All media were autoclaved at $121^{\circ} \mathrm{C}$ for 30 min before use.

\section{Ion implantation}

B. amyloliquefaciens spores suspended in liquid were removed aseptically from cultures of the parental RL strain grown in slant store culture medium for $72 \mathrm{~h}$ at $37^{\circ} \mathrm{C}$. Next, 0.2 $\mathrm{mL}$ of the spore suspension was spread on an empty culture dish. The spores were air-dried at room temperature and irradiated with an LZD900 multifunctional implantation apparatus equipped with an ion-beam bioengineering instrument (Institute of Plasma Physics, Chinese Academy of Sciences). The spores were implanted with $\mathrm{N}^{+}$or $\mathrm{Ar}^{+}$ions at $10 \mathrm{keV}$, with doses of $0.52 \times 10^{15}, 1.04 \times 10^{15}, 1.56 \times 10^{15}, 2.08 \times 10^{15}, 2.6 \times 10^{15}$, and $3.12 \times 10^{15} \mathrm{ions} / \mathrm{cm}^{2}$. Briefly, the target chamber was evacuated to $1 \times 10^{-3} \mathrm{~Pa}$. The parental control spores were only exposed to the vacuum, but the experimental spores were exposed to 5-s pulses of implantation every $50 \mathrm{~s}$. Next, the spores were washed aseptically with water and incubated at $37^{\circ} \mathrm{C}$ for $1-2$ days. The cells were then prepared for screening after slant culture for 3 days.

\section{Mutant screening}

The monospores from the samples subjected to ion implantation were washed using $1 \mathrm{~mL}$ sterile water, and $0.1 \mathrm{~mL}$ of various dilutions of the monospore suspension was spread onto 3-5 culture dishes containing preliminary screening culture medium. The colonies were then incubated at $41.5^{\circ} \mathrm{C}$ for $36 \mathrm{~h}$. Single colonies were selected on the basis of the greater ratio of the diameter of the colony to that of the transparent circle. The enzymatic activities of the selected mutant strains and the RL parental strain were tested by culturing in 250 -mL flasks (containing the shaking flask culture medium) at $41.5^{\circ} \mathrm{C}$ for $48 \mathrm{~h}$. The enzymatic activity of the mutant thermostable $\alpha$-amylase was measured using a spectrophotometric method (Azad et al., 2009). 


\section{RESULTS}

\section{Dose effects of $\mathrm{N}^{+}$and $\mathrm{Ar}^{+}$implantation on the RL strain}

To calculate the survival rate, the RL strain was implanted with different doses of either $\mathrm{N}^{+}$or $\mathrm{Ar}^{+}$at $10 \mathrm{keV}$. As shown in Figure 1, the survival rate of the strain decreased rapidly as the ion implantation dose increased to $1.5 \times 10^{15} \mathrm{ions} / \mathrm{cm}^{2}$. The survival rate increased slightly at doses of 1.5 and $2.0 \times 10^{15}$ ions $/ \mathrm{cm}^{2}$. However, the survival rate declined as the implantation dose increased beyond $2.08 \times 10^{15} \mathrm{ions} / \mathrm{cm}^{2}$. This phenomenon is described as a saddle. Although $\mathrm{N}^{+}$and $\mathrm{Ar}^{+}$implantations resulted in similar survival trends in RL, lethality was slightly greater in $\mathrm{Ar}^{+}$than in $\mathrm{N}^{+}$at the same doses.

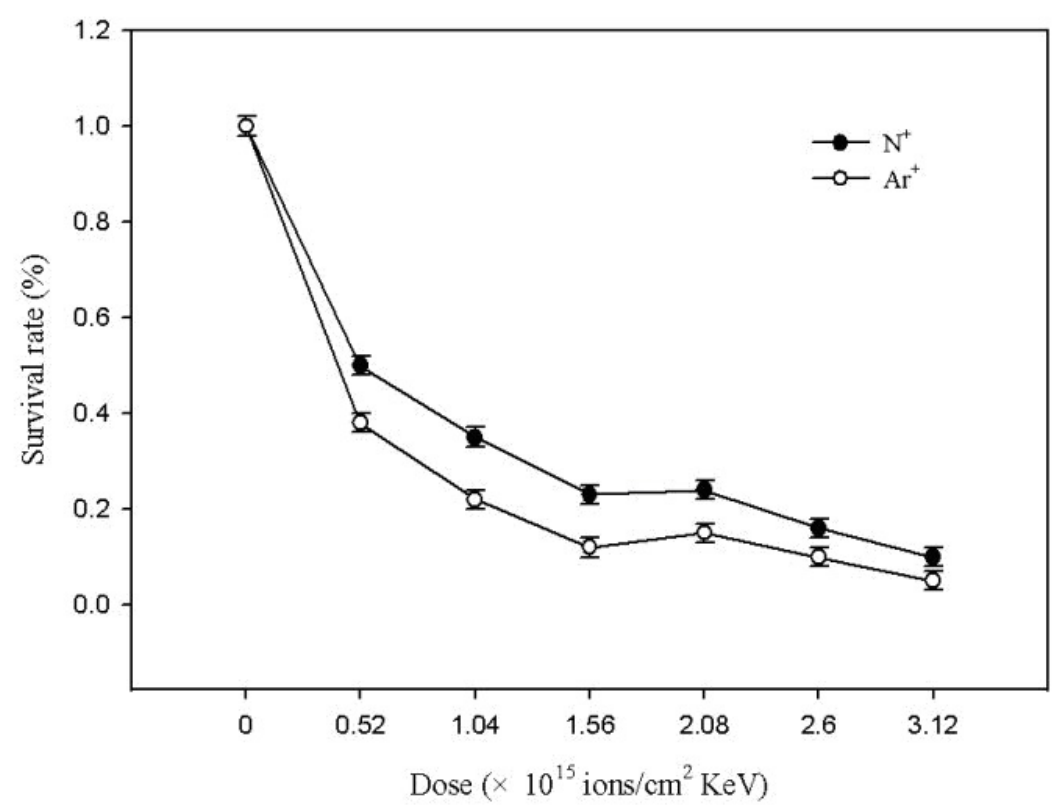

Figure 1. Survival rate of RL after $\mathrm{N}^{+}$or $\mathrm{Ar}^{+}$implantation.

Enzymatic activity was measured in liquid fermentation cultures of the mutant strains; 3 independent experiments were performed with consistent results. Mutant cultures with an absorbance at $395 \mathrm{~nm}\left(\mathrm{~A}_{395}\right) 10 \%$ higher or lower than that of the parental strain were defined as positive or negative mutants, respectively, whereas those with an $A_{395}$ within $10 \%$ of the control were defined as insignificant.

The results shown in Figures 2 and 3 demonstrate that the mutation rate (calculated as the enzymatic activity of $\alpha$-amylase) increased with increasing $\mathrm{N}^{+}$or $\mathrm{Ar}^{+}$implantation doses. The maximum mutation rate was obtained with a dose of $2.08 \times 10^{15} \mathrm{ions} / \mathrm{cm}^{2}$. In addition, the mutation rate caused by $\mathrm{N}^{+}$implantation was greater than that caused by $\mathrm{Ar}^{+}$implantation; this finding highlighted that mutation effects differ and depend on both the dose and type of implanted ions. On the basis of these results, an ion implantation dose of $2.08 \times 10^{15} \mathrm{ions} / \mathrm{cm}^{2}$ at $10 \mathrm{keV}$ was used in the following implantation experiments. 


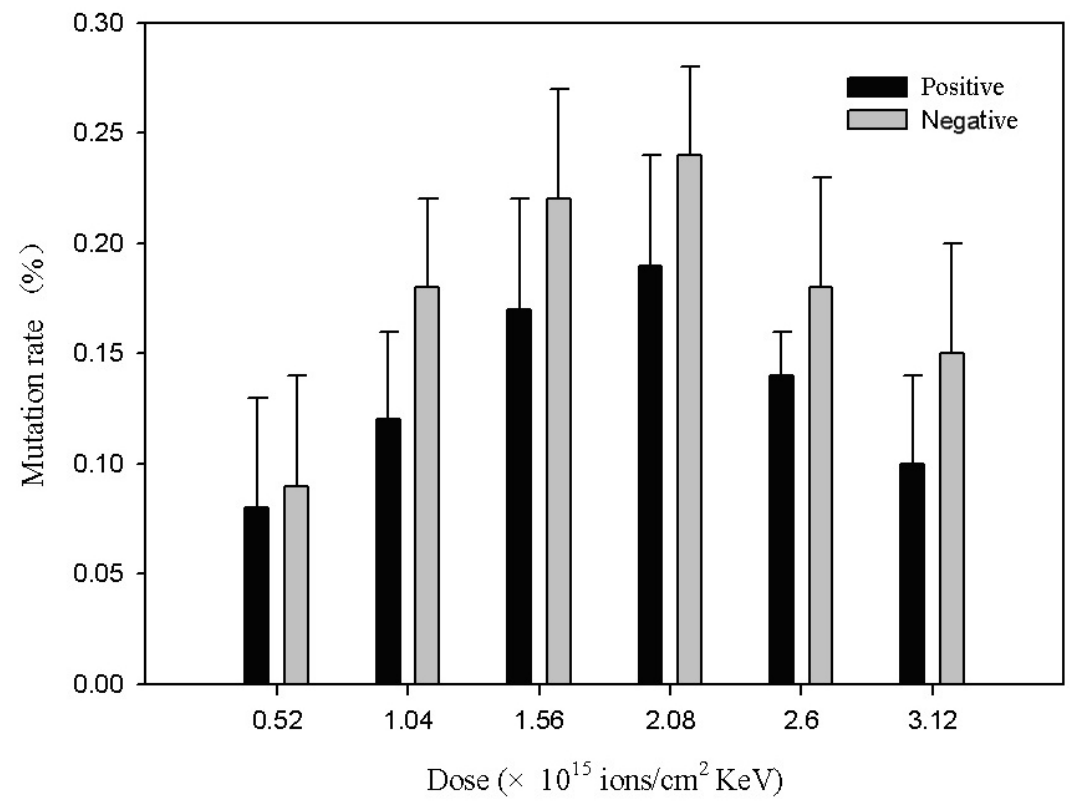

Figure 2. Mutation rates of RL implanted with $\mathrm{N}^{+}$at $10 \mathrm{keV}$. A positive value represents a $10 \%$ higher mutation rate in the mutant than in the parental strain; a negative value represents a $10 \%$ lower mutation rate in the mutant than in the parental strain.

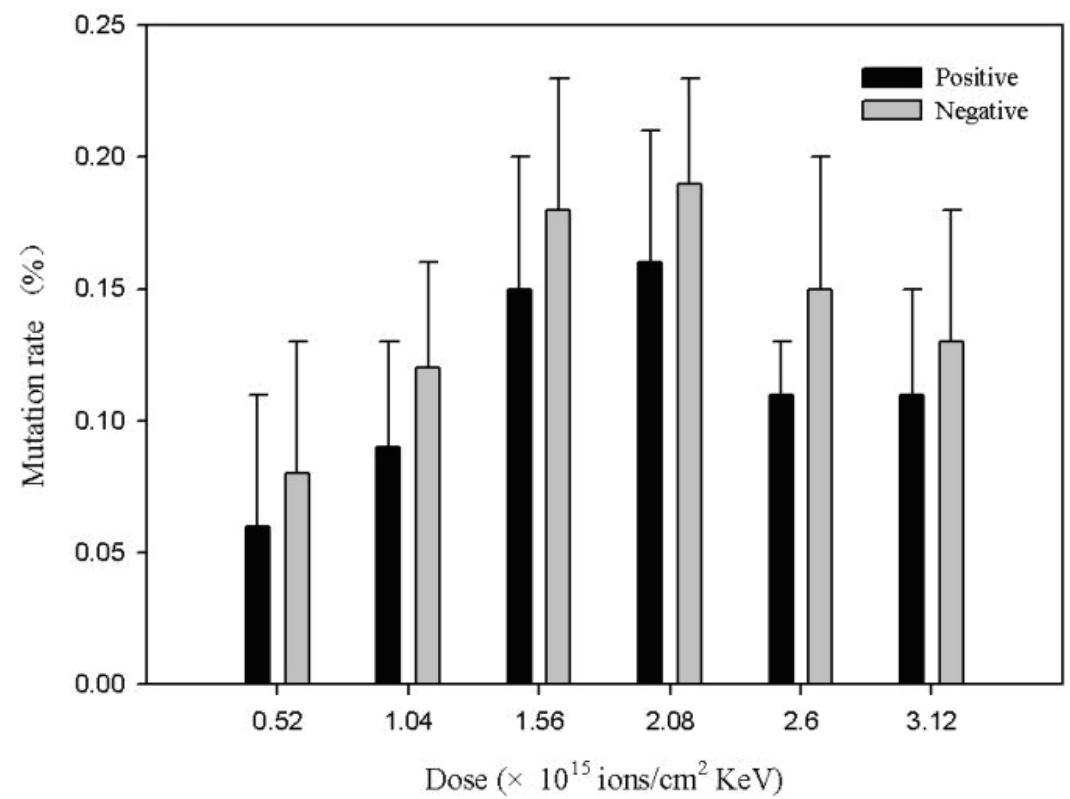

Figure 3. Mutation rates of RL implanted with $\mathrm{Ar}^{+}$at $10 \mathrm{keV}$. A positive value represents a $10 \%$ higher mutation rate in the mutant than in the parental strain; a negative value represents a $10 \%$ lower mutation rate in the mutant than in the parental strain. 


\section{Screening for highly active and thermostable $\alpha$-amylase strains}

$\mathrm{N}^{+}$ion implantation was used to mutate the parent RL strain as described above, and the mutants were screened for altered $\alpha$-amylase activity. Of the colonies screened, 27 colonies had a larger halo diameter than the control. Further testing revealed that enzymatic activity in 6 of these mutants (RL-1, $-2,-3,-4,-5$, and -6 ) was constantly and significantly higher than that of the RL strain. The results shown in Table 1 indicate that the $\alpha$-amylase activity and time for optimal enzyme activity varied among the 6 mutants. The greatest increase in enzymatic activity was found in the mutant strain RL-1 (57.1\% greater than RL), in which the time required to reach optimal enzyme activity was decreased by approximately $4 \mathrm{~h}$ relative to the parental strain. When the strains were cultured under the same conditions, the starch degradation halo caused by RL-1 was visibly larger than that of the parental strain (Figure 4). Thus, the relative $\alpha$-amylase activity of RL-1 was inferred to be greater than that of RL.

Table 1. Results of the mutant screen.
\begin{tabular}{lccc}
\hline Strain & Highest enzyme activity (U/mL) & Time to optimal enzyme activity (h) & $\begin{array}{c}\text { Increased activity of mutant compared } \\
\text { to the parental strain }(\%)\end{array}$ \\
\hline RL & $37.26 \pm 0.09$ & $40.5 \pm 0.5$ & - \\
RL-1 & $58.51 \pm 0.12^{*}$ & $36.6 \pm 0.4$ & 57.1 \\
RL-2 & $45.31 \pm 0.07^{*}$ & $48.3 \pm 0.6$ & 21.6 \\
RL-3 & $50.60 \pm 0.09^{*}$ & $36.5 \pm 0.7$ & 35.8 \\
RL-4 & $52.17 \pm 0.10^{*}$ & $54.4 \pm 0.5$ & 53.4 \\
RL-5 & $41.62 \pm 0.06^{*}$ & $48.7 \pm 0.4$ & 11.7 \\
RL-6 & $39.20 \pm 0.09$ & $48.3 \pm 0.3$ & 5.2 \\
\hline
\end{tabular}

*Enzyme activity of the mutant was higher than that of the parent strain with statistical significance $(\mathrm{P}<0.05)$.

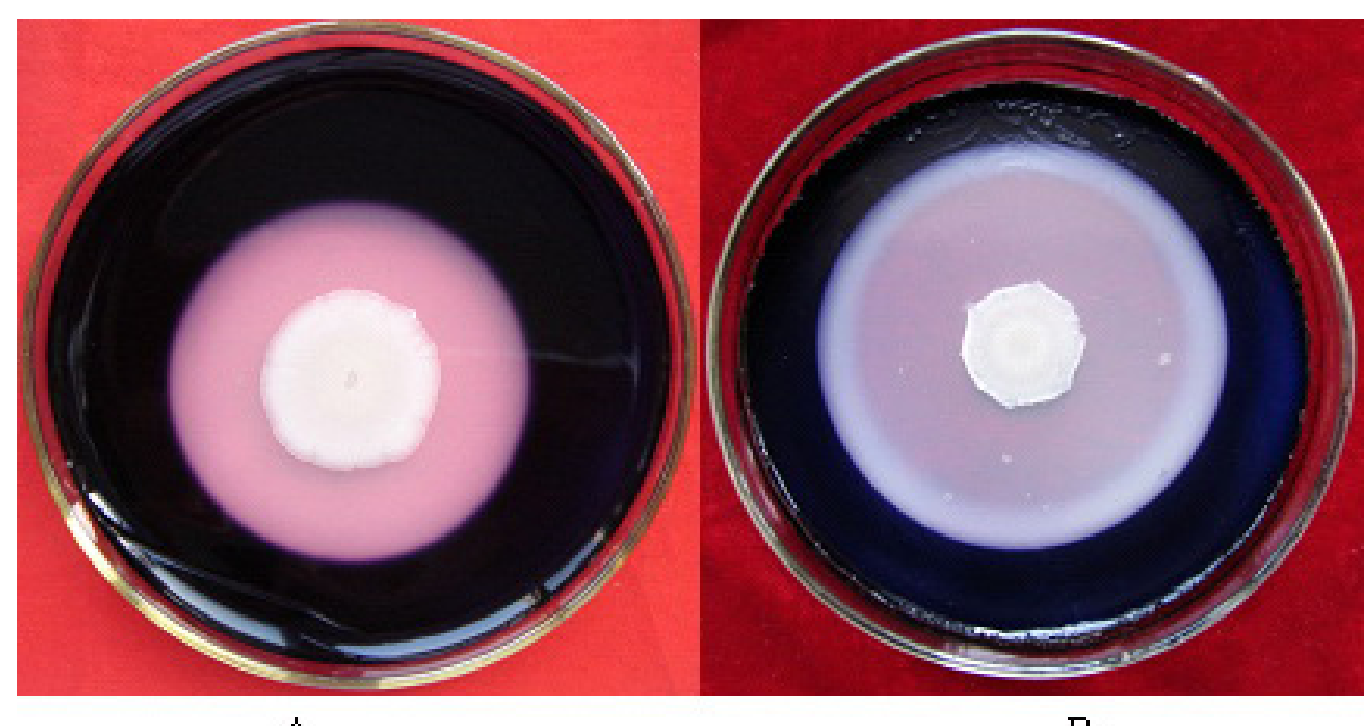

A

$\mathrm{B}$

Figure 4. Starch degradation by RL (A) and RL-1 (B). 


\section{Genetic stability of the RL-1 mutation}

To determine the genetic stability of the $\alpha$-amylase mutation(s), RL-1 was subcultured on solid media for 8 generations. As shown in Table 2, the enzymatic activity of the thermostable $\alpha$-amylase in RL-1 was highly genetically stable. The mutant strain retained high enzyme activity even after culturing for 8 generations.

\begin{tabular}{|c|c|}
\hline Generation & $\alpha$-Amylase activity $(\mathrm{U} / \mathrm{mL})^{\mathrm{a}}$ \\
\hline 1 & $58.5 \pm 0.11$ \\
\hline 2 & $58.2 \pm 0.08$ \\
\hline 3 & $58.7 \pm 0.09$ \\
\hline 4 & $57.9 \pm 0.07$ \\
\hline 5 & $58.3 \pm 0.12$ \\
\hline 6 & $58.1 \pm 0.09$ \\
\hline 7 & $57.5 \pm 0.10$ \\
\hline 8 & $58.5 \pm 0.13$ \\
\hline
\end{tabular}

${ }^{a}$ Results are pooled from three independent experiments.

\section{Identification of the extracellular enzyme}

After culturing RL-1 in a flask with vigorous shaking for $24 \mathrm{~h}$, the supernatant and cells were separated by centrifugation at $6000 \mathrm{rpm}$ for $10 \mathrm{~min}$. The RL-1 cells were lysed to allow for the measurement of the activities of enzymes in the cell supernatant and fermentation solution. Our results indicated that both the supernatant and fermentation solution contain $\alpha$-enzyme activities that were largely lacking in the RL-1 cells. This indicates that the $\alpha$-enzyme was secreted by RL-1 into the fermentation solution.

\section{DISCUSSION}

The sensitivity of various microorganisms to ion beams is different. B. amyloliquefaciens has been widely used in industry, agriculture, environmental reclamation, pest control, etc. (Machius et al., 1995; Igarashi et al., 1998; Asghari et al., 2004; Shareghi et al., 2007). In the past, X-rays and gamma rays were mainly used to mutate microorganisms; however, repeated use of these methods has led to an increase in the resistance of microbes, decrease in mutation efficiency, and narrowing of the mutation spectrum. To avoid such issues, low-energy ion implantation has been utilized as a novel mutation source, but the application of this technology has been very limited in B. amyloliquefaciens compared to Escherichia coli. As seen from the survival curves (Figure 1) and the amylase mutation curves (Figures 2 and 3 ) in this study, the B. amyloliquefaciens RL strain is sensitive to low-energy ions. However, the survival curve of RL is similar to that of E. coli (Fondy et al., 1989); both resemble saddles. Additionally, implantation of RL with different doses of $\mathrm{N}^{+}$and $\mathrm{Ar}^{+}$demonstrated that the $\alpha$-amylase mutation rate of $\mathrm{N}^{+}$was higher than that of $\mathrm{Ar}^{+}$, perhaps due to decreased cell damage caused by $\mathrm{N}^{+}$implantation. Interestingly, these same trends were also reported in E. coli and cotton.

The effects of low-energy ions depend on the type and radiation mass of the ion. The difference between ion beams and X- or gamma rays is that low-energy ions have larger energy, mass, and momentum effects. Ions with more mass have larger mass and momentum 
effects, and therefore, they induce greater damage to the microbes. Indeed, in our experiments, the survival rate of recipients was lower than that of controls. However, different ions have varying mutational effects. For example, nitrogen is one of several important elements that form the nucleotide bases of DNA, and $\mathrm{N}^{+}$implantation can directly cause molecular structural changes in these bases. This can increase the DNA mismatch rate, which would manifest as an increased mutation rate during DNA replication. In contrast, $\mathrm{Ar}^{+}$is more likely to cause heavy damage via single- and double-strand breaks in DNA. The in vitro and in vivo effects of various ions on DNA breakage and mutation have been previously confirmed (Nordhoff et al., 1993; Sandstrom et al., 1994; Kiefer et al., 2002; Liu et al., 2003), but our results suggest that $\mathrm{N}^{+}$is the optimal ion for inducing mutations in microorganisms.

A highly active, thermostable $\alpha$-amylase would be beneficial to the fermentation industry. In the present study, we used low-energy $\mathrm{N}^{+}$ion implantation to mutate the B. amyloliquefaciens strain RL, an important microbe (with roles in many aspects of industry, agriculture, environmental reclamation, pest control, etc.) that secretes a thermostable $\alpha$-amylase, and screened for mutants with increased starch degradation activity (Declerck et al., 2000; Kim et al., 2003; Du et al., 2006; Khemakhem et al., 2009). We found that, compared to the parental strain, the mutant strain RL-1 excreted a highly thermostable $\alpha$-amylase with $57.1 \%$ increased activity $(58.51 \mathrm{U} / \mathrm{mL})$.

The results reported in this paper demonstrate that low-energy ion implantation is a feasible mutation technique in $B$. amyloliquefaciens and that this technique could be used for improving the enzymatic activity of $\alpha$-amylase. However, the mechanism underlying the increase in activity of this thermostable $\alpha$-amylase requires further study.

\section{ACKNOWLEDGMENTS}

Research supported by the National Key Project of Transgenic Crops of China (\#2008ZX08010-001) and National Natural Science Foundation of China (\#30771309 and \#11075001).

\section{REFERENCES}

Asghari SM, Khajeh K, Ranjbar B, Sajedi RH, et al. (2004). Comparative studies on trifluoroethanol (TFE) state of a thermophilic alpha-amylase and its mesophilic counterpart: limited proteolysis, conformational analysis, aggregation and reactivation of the enzymes. Int. J. Biol. Macromol. 34: 173-179.

Azad MA, Bae JH, Kim JS, Lim JK, et al. (2009). Isolation and characterization of a novel thermostable alpha-amylase from Korean pine seeds. N. Biotechnol. 26: 143-149.

Declerck N, Machius M, Wiegand G, Huber R, et al. (2000). Probing structural determinants specifying high thermostability in Bacillus licheniformis alpha-amylase. J. Mol. Biol. 301: 1041-1057.

Dong Y, Liu Y, Chen Y, Niu D, et al. (2008). Purification and characterization of thermostable amylases from two bacterial species. Wei Sheng Wu Xue Bao 48: 169-175.

Du BB, Hao S, Li YM, Yue LL, et al. (2006). Expression of a thermostable a-amylase mutant into Escherichia coli and Pichia pastoris. Wei Sheng Wu Xue Bao 46: 827-830.

Fielden MR, Matthews JB, Fertuck KC, Halgren RG, et al. (2002). In silico approaches to mechanistic and predictive toxicology: an introduction to bioinformatics for toxicologists. Crit. Rev. Toxicol. 32: 67-112.

Fondy BR, Geiger DR and Servaites JC (1989). Photosynthesis, carbohydrate metabolism, and export in Beta vulgaris L. and Phaseolus vulgaris L. during square and sinusoidal light regimes. Plant Physiol. 89: 396-402.

Hoj PB, Hartman DJ, Morrice NA, Doan DN, et al. (1989). Purification of (1 $\rightarrow 3)$-beta-glucan endohydrolase isoenzyme II from germinated barley and determination of its primary structure from a cDNA clone. Plant Mol. Biol. 13: 31-42. 
Igarashi K, Hatada Y, Hagihara H, Saeki K, et al. (1998). Enzymatic properties of a novel liquefying alpha-amylase from an alkaliphilic Bacillus isolate and entire nucleotide and amino acid sequences. Appl. Environ. Microbiol. 64: 32823289 .

Khemakhem B, Ali MB, Aghajari N, Juy M, et al. (2009). Engineering of the alpha-amylase from Geobacillus stearothermophilus US100 for detergent incorporation. Biotechnol. Bioeng. 102: 380-389.

Kiefer J, Egenolf R and Ikpeme S (2002). Heavy ion-induced DNA double-strand breaks in yeast. Radiat. Res. 157: 141148.

Kim YW, Choi JH, Kim JW, Park C, et al. (2003). Directed evolution of Thermus maltogenic amylase toward enhanced thermal resistance. Appl. Environ. Microbiol. 69: 4866-4874.

Li M, Wu YJ, Yu ZL, Sheng GP, et al. (2009). Enhanced nitrogen and phosphorus removal from eutrophic lake water by Ipomoea aquatica with low-energy ion implantation. Water Res. 43: 1247-1256.

Liu J, Li Q, Yu Y and Fang X (2003). Spectroscopic and electrochemical studies of DNA breakage induced by dopamine and copper ion. Anal. Sci. 19: 1099-1102.

Machius M, Wiegand G and Huber R (1995). Crystal structure of calcium-depleted Bacillus licheniformis alpha-amylase at 2.2 A resolution. J. Mol. Biol. 246: 545-559.

Mollania N, Khajeh K, Hosseinkhani S and Dabirmanesh B (2010). Purification and characterization of a thermostable phytate resistant alpha-amylase from Geobacillus sp. LH8. Int. J. Biol. Macromol. 46: 27-36.

Nordhoff E, Cramer R, Karas M, Hillenkamp F, et al. (1993). Ion stability of nucleic acids in infrared matrix-assisted laser desorption/ionization mass spectrometry. Nucleic Acids Res. 21: 3347-3357.

Okita TW, Greenberg E, Kuhn DN and Preiss J (1979). Subcellular localization of the starch degradative and biosynthetic enzymes of spinach leaves. Plant Physiol. 64: 187-192.

Sandstrom BE, Granstrom M and Marklund SL (1994). New roles for quin2: powerful transition-metal ion chelator that inhibits copper-, but potentiates iron-driven, Fenton-type reactions. Free Radic. Biol. Med. 16: 177-185.

Shareghi B, Arabi M and Zargham M (2007). Denaturation of Bacillus amyloliquefaciens alpha-amylase with urea. Pak. J. Biol. Sci. 10: 3154-3157.

Tee BL and Kaletunc G (2009). Immobilization of a thermostable alpha-amylase by covalent binding to an alginate matrix increases high temperature usability. Biotechnol. Prog. 25: 436-445.

Xie C, Yao J, Pan R, Wu L, et al. (2003). Mutagenesis of ion beam implantation and identification of two new rifampicin resistance determining sites in rpoB gene in Escherichia coli. Wei Sheng Wu Xue Bao 43: 732-739.

Yamate N and Yamazaki T (1999). Is the difference in alpha-amylase activity in the strains of Drosophila melanogaster with different allozymes due to transcriptional or posttranscriptional control? Biochem. Genet. 37: 345-356. 